\title{
Acquired resistance to temsirolimus in human renal cell carcinoma cells is mediated by the constitutive activation of signal transduction pathways through mTORC2
}

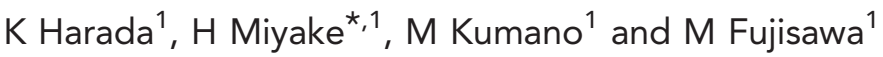 \\ ${ }^{1}$ Division of Urology, Kobe University Graduate School of Medicine, Chuo-ku, Kobe 650-0017, Japan
}

Background: The objective of this study was to characterise the mechanism underlying acquired resistance to temsirolimus, an inhibitor of mammalian target of rapamycin (mTOR), in renal cell carcinoma (RCC).

Methods: A parental human RCC cell line, ACHN (ACHN/P), was continuously exposed to increasing doses of up to $20 \mu \mathrm{m}$ of temsirolimus, and a cell line resistant to temsirolimus (ACHN/R), showing a sixfold higher $\mathrm{IC}_{50}$ than that of $A C H N / P$, was developed.

Results: Following treatment with temsirolimus, phosphorylation of S6 kinase in ACHN/P was markedly inhibited, whereas there was no detectable expression of phosphorylated S6 in ACHN/R before and after temsirolimus treatment. However, AKT and p44/42 mitogen-activated protein kinase (MAPK) were constitutively phosphorylated even after temsirolimus treatment in $A C H N / R$, but not in ACHN/P. There was no significant difference between the sensitivities of ACHN/P and ACHN/R to KU0063794, a dual inhibitor of mTOR complex 1 (mTORC1) and mTORC2. Similar sensitivities to temsirolimus in ACHN/P and ACHN/R could be achieved by additional treatment with specific inhibitors of AKT- and MAPK-signaling pathways.

Conclusion: The activation of signal transduction pathways via mTORC2, but not via mTORC1, may have an important role in the acquisition of a resistant phenotype to temsirolimus in RCC.

Renal cell carcinoma (RCC) is the most common malignant tumour arising in the adult kidney, and annual estimates of newly diagnosed patients have been steadily increasing. One of the most unique characteristics of RCC is the high incidence of metastatic spread to distant organs (Rini et al, 2008). Owing to a phenotype that is highly resistant to conventional chemotherapeutic agents, immunotherapy using cytokines had been performed for patients with metastatic RCC as first-line therapy, which resulted in limited efficacy, with an objective response rate of less than $20 \%$; therefore, the prognosis of patients with metastatic RCC has been generally poor, with a median overall survival of approximately 1 year (Parton et al, 2006; Miyake et al, 2009). In recent years, however, novel molecular targeted drugs have been developed based on the elucidation of molecular mechanisms mediating the progression of RCC, and a marked paradigm shift in the therapeutic strategy for metastatic RCC has occurred following the introduction of these new agents into clinical practice (Bellmunt et al, 2007; Lane et al, 2007).

The mammalian target of rapamycin (mTOR), a highly conserved serine/threonine kinase, functions as an important regulator of a wide variety of cellular functions, such as cell proliferation, apoptosis and angiogenesis, by controlling cellular catabolism and anabolism. The mTOR protein forms two unique complexes-mTOR complex 1 (mTORC1) and mTORC2 (Menon and Manning, 2008). The mTORC1 function was shown to be mediated by the phosphorylation of $\mathrm{S} 6 \mathrm{~K} 1$ and $4 \mathrm{E}-\mathrm{BP} 1$, which 
stimulate protein synthesis by mRNA translation and cell growth by entering the G1 phase of the cell cycle (Foster and Fingar, 2010), whereas mTORC2, initially identified as a regulator of the actin cytoskeleton, has been shown to phosphorylate members of the AGC kinase family, including Akt, which is linked to several pathological conditions (Sparks and Guertin, 2010). Considering these findings, mTOR is currently regarded as a potential therapeutic target for various types of malignancies, including RCC.

Temsirolimus, a rapamycin analogue, is one of the most widely used mTOR inhibitors against metastatic RCC (Voss et al, 2011). In preclinical RCC models, temsirolimus has been shown to exhibit an inhibitory effect on the growth of a wide variety of tumour cells through the inactivation of key signal transduction pathways regulating the cell cycle and angiogenesis (Le Tourneau et al, 2008). In a clinical setting, an international randomised phase III trial (Global Advanced RCC trial) that included patients with previously untreated poor-prognosis metastatic RCC, demonstrated significant overall as well as progression-free survival benefits of temsirolimus compared with interferon- $\alpha$ (Hudes et al, 2007). However, the acquisition of a phenotype that is resistant to this agent is of major clinical concern; that is, the vast majority of patients with metastatic RCC showing an initial favourable response to temsirolimus eventually show disease progression (Rini and Atkins, 2009). Although there have been several hypotheses on the molecular mechanism involved in the acquisition of resistance to mTOR inhibitors, including temsirolimus, by cancer cells (Carew et al, 2011), the mechanism remains largely unknown.

In this study, we established a human RCC cell line that is resistant to temsirolimus and investigated changes in molecular profiles in this cell line in order to identify potential targets for overcoming acquired resistance to this agent.

\section{MATERIALS AND METHODS}

Tumour cell line. ACHN, derived from human RCC, was purchased from the American Type Culture Collection (Rockville, MD, USA) and used within 6 months of resuscitation. Cells were maintained in MEM (Life Technologies Inc., Gaithersburg, $\mathrm{MD}, \mathrm{USA}$ ) and supplemented with $5 \%$ heat-inactivated fetal bovine serum.

The RCC cells resistant to temsirolimus were generated by growing parental ACHN (ACHN/P) cells serially treated with an increasing dose of up to $20 \mu \mathrm{M}$ of temsirolimus (Cayman Chemical, Ann Arbor, MI, USA). Following continuous culture in complete medium supplemented with $20 \mu \mathrm{M}$ of temsirolimus for more than 20 passages, these cells were used as a temsirolimus-resistant RCC cell line $(\mathrm{ACHN} / \mathrm{R})$ for all subsequent experiments.

Cell proliferation assay. To compare the in vitro proliferation of ACHN sublines (ACHN/P and ACHN/R), $5 \times 10^{4}$ cells of each cell line were seeded in each well of 12 -well plates and allowed to attach overnight, and the number of cells in each cell line was assessed using Cell Counting Kit-8 (Dojindo Molecular Technologies, Kumamoto, Japan). In addition, the effects of treatment with temsirolimus (Sigma-Aldrich, St Louis, MO, USA), sunitinib, sorafenib (Toronto Research Chemicals, Toronto, Canada), everolimus (LC Laboratories, Woburn, MA, USA) or KU0063794 (Wako, Tokyo, Japan) in combination with LY294002 or U0126 (Wako) on the proliferation of ACHN sublines were also examined after $48 \mathrm{~h}$ of incubation with these agents. Each assay was performed in triplicate.

Western blot analysis. Western blot analysis was performed as described previously (Kumano et al, 2008). Samples containing equal amounts of protein $(25 \mu \mathrm{g})$ from lysates of the ACHN sublines cultured in either a standard medium or one containing $10 \mu \mathrm{M}$ of temsirolimus were subjected to SDS-polyacrylamide gel electrophoresis and transferred to a nitrocellulose filter. The filter was blocked using PBS containing $5 \%$ nonfat milk powder at $4{ }^{\circ} \mathrm{C}$ overnight and then incubated for $1 \mathrm{~h}$ with antibodies against Bcl-2, Bcl-xL (Santa Cruz Biotechnology, Santa Cruz, CA, USA), Bax (EPITOMICS, Burlingame, CA, USA), clusterin (Santa Cruz Biotechnology), raptor, rictor (EPITOMICS), total and phosphorylated S6 (Cell Signaling Technology, Danvers, MA, USA), $\beta$-actin (Santa Cruz Biotechnology), total and phosphorylated Akt, p44/42 mitogen-activated protein kinase (MAPK) and signal transducers and activation of transcription 3 (STAT3) (Cell Signaling Technology). The filters were then incubated for $30 \mathrm{~min}$ with horseradish-peroxide-conjugated secondary antibodies (Santa Cruz Biotechnology), and specific proteins were detected using an enhanced chemiluminescence Western blot analysis system (Amersham, Life Science, Arlington Heights, IL, USA). The strength of each signal density was determined using a densitometer (Bio-Tek Instruments, Inc., Winooski, VT, USA).

Assessment of in vivo tumour growth. Male athymic nude mice (BALB/c-nu/nu males, 5-6 weeks old) were purchased from Clea Japan (Tokyo, Japan) and housed in a controlled environment at $22^{\circ} \mathrm{C}$ on a 12 -h light, 12 -h dark cycle. Animal experiments were conducted in accordance with the National Institutes of Health Guide for the Care and Use of Laboratory Animals. Each experimental group consisted of 10 mice. The tumour cells of each ACHN subline were trypsinised, washed twice with PBS, and $5 \times 10^{6}$ cells were subcutaneously injected with $100 \mu$ l of Matrigel (Becton Dickinson, Franklin Lakes, NJ, USA). In the first set of experiments, 3 weeks after the implantation of tumour cells, mice were randomly selected for the intraperitoneal administration of either temsirolimus at a dose of $10 \mathrm{mg} \mathrm{kg}^{-1}$ or of the vehicle three times per week for 4 weeks. In the second set of experiments, mice were treated with KU0063794 at a dosage of $8 \mathrm{mg} \mathrm{kg}^{-1}$ twice per week for 4 weeks. Subcutaneous tumour growth was measured using calipers and calculated as previously described (Terakawa et al, 2009).

Statistical analysis. Differences between the two groups were compared using the unpaired $t$-test. All statistical calculations were performed using the Statview 5.0 software (Abacus Concepts, Inc., Berkley, CA, USA), and $P$ values $<0.05$ were considered significant.

\section{RESULTS}

Comparison of sensitivities to several molecular targeted agents between ACHN sublines. ACHN/R cells were generated by the continuous exposure of growing $\mathrm{ACHN} / \mathrm{P}$ cells to increasing doses of up to a final concentration of $20 \mu \mathrm{M}$ of temsirolimus. There was no significant difference between the growth patterns of ACHN/P and ACHN/R cells when cultured in a standard medium without temsirolimus (data not shown). However, ACHN-R cells exhibited significantly higher resistance to treatment with temsirolimus than did $\mathrm{ACHN} / \mathrm{P}$ cells, showing an approximately sixfold higher $\mathrm{IC}_{50}$ (Figure 1).

It was subsequently examined whether ACHN/R cells acquire cross-resistance to several molecular targeted agents against advanced RCC other than temsirolimus. As shown in Figure 2A, ACHN/R cells were cross-resistant to everolimus, another potential inhibitor of mTOR, with an approximately sixfold higher $\mathrm{IC}_{50}$ than that of $\mathrm{ACHN} / \mathrm{P}$ cells. However, there were no significant differences between the ACHN sublines in sensitivity to multiple tyrosine kinase inhibitors (TKIs), sorafenib and sunitinib (Figure $2 \mathrm{~B}$ and $\mathrm{C}$ ). 
Expression of key molecules associated with apoptosis in ACHN sublines. Western blot analyses were used to determine whether the acquisition of a phenotype that is resistant to temsirolimus by ACHN/P cells induces changes in the expression of major molecules involved in apoptosis. As shown in Figure 3, treatment with temsirolimus resulted in an increase in the expression of $\mathrm{Bcl}-2$ in $\mathrm{ACHN} / \mathrm{R}$ cells, but not in $\mathrm{ACHN} / \mathrm{P}$ cells, whereas Bax expression was upregulated in $\mathrm{ACHN} / \mathrm{P}$ cells, but not in ACHN/R cells, following treatment with temsirolimus. However, there were no significant differences in the expression levels of Bcl-xL and clusterin between ACHN sublines before and after temsirolimus treatment.

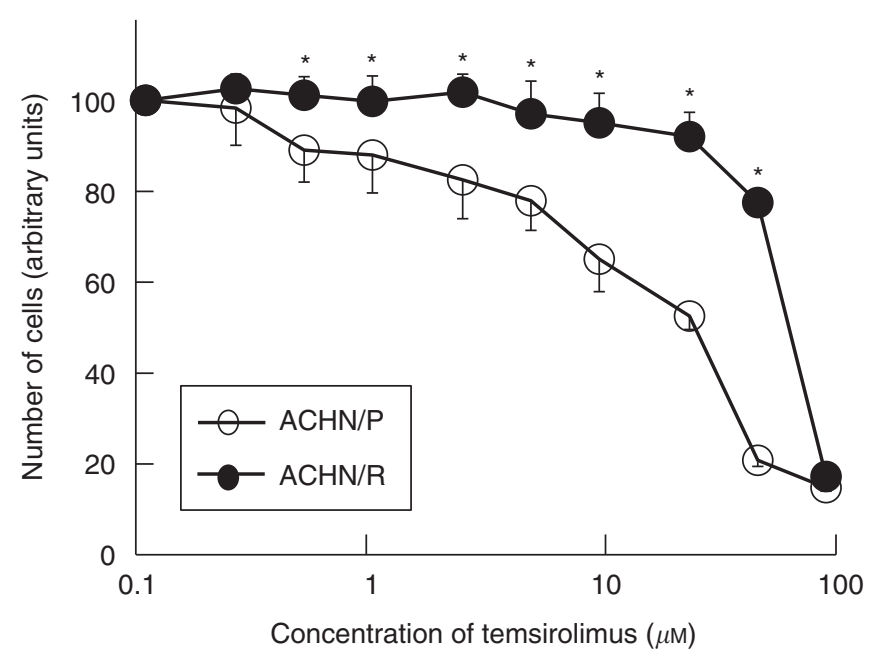

Figure 1. Effect of treatment with temsirolimus on the in vitro cell growth of ACHN sublines. The ACHN/P and ACHN/R cells were treated with the indicated doses of temsirolimus. After $48 \mathrm{~h}$ of incubation, cell growth was determined in triplicate in three independent experiments. Bars, s.d. * ${ }^{*}$ differs from ACHN/P $(P<0.01)$.
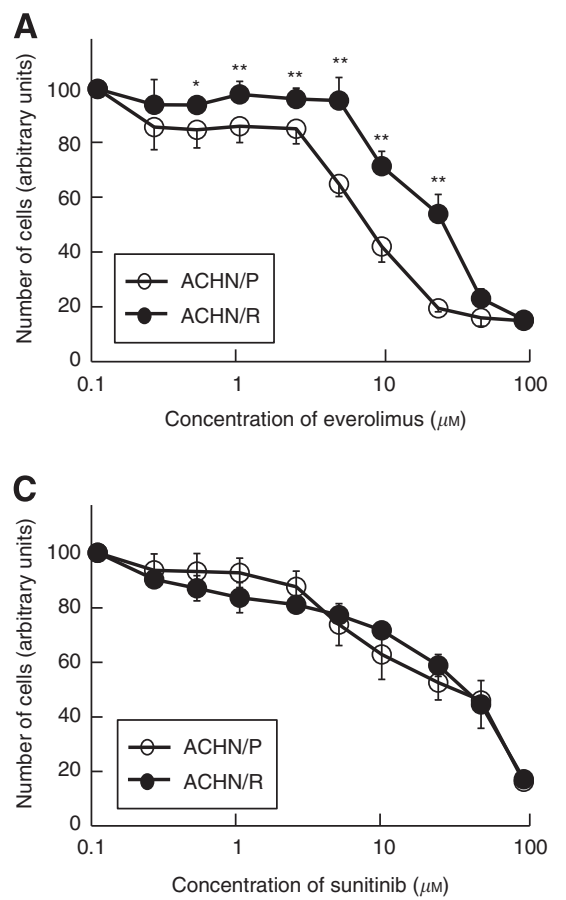

Different activation statuses of major signal transduction pathways between ACHN sublines. Changes in signal transduction pathways in $\mathrm{ACHN}$ sublines following temsirolimus treatment were investigated. There were no significant differences in the expression levels of raptor and rictor, which delineate mTORC1 and mTORC2, respectively, between ACHN sublines. Furthermore, despite the abundant expression of phosphorylated S6 in $\mathrm{ACHN} / \mathrm{P}$ cells prior to treatment with temsirolimus, treatment of ACHN/P cells with temsirolimus resulted in the complete inhibition of phosphorylated S6 expression, whereas the S6 pathway in ACHN/R cells appeared to be completely inactivated irrespective of temsirolimus treatment; that is, detectable

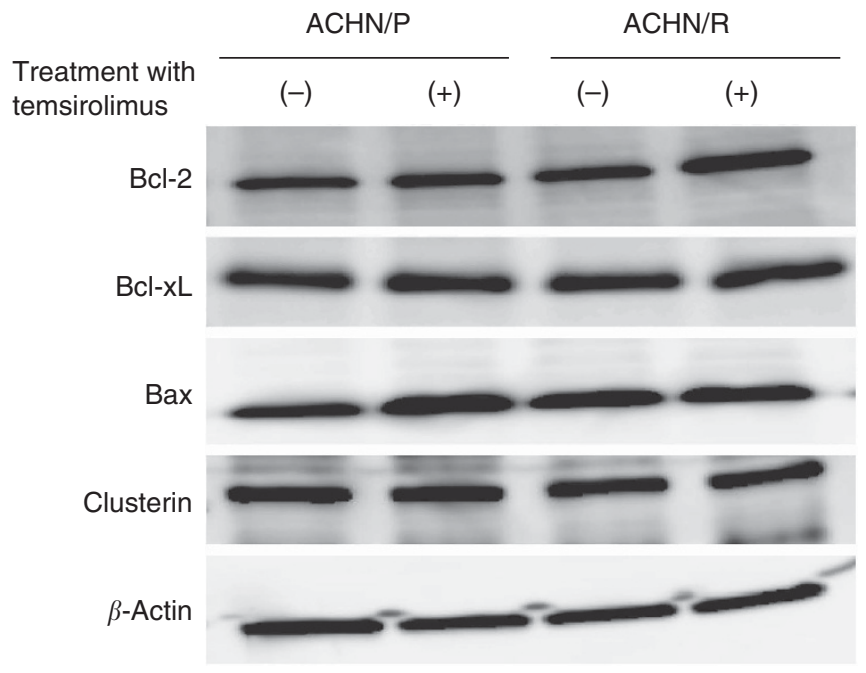

Figure 3. Changes in expression patterns of key molecules involved in apoptosis in $\mathrm{ACHN}$ sublines following treatment with temsirolimus. Expression levels of $\mathrm{Bcl}-2, \mathrm{Bcl}-\mathrm{xL}, \mathrm{Bax}$, clusterin and $\beta$-actin in $\mathrm{ACHN}$ sublines before and after treatment with temsirolimus $(10 \mu \mathrm{m})$ were analysed using western blotting.

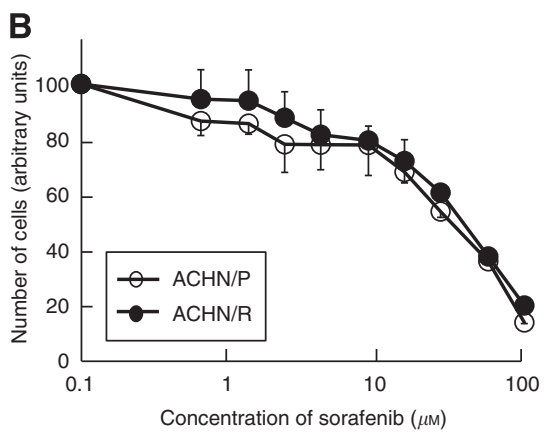

Figure 2. Effects of treatment with several molecular targeted agents on the in vitro cell growth of $A C H N$ sublines. The ACHN/P and ACHN/R cells were treated with the indicated doses of (A) everolimus, (B) sorafenib or (C) sunitinib. After $48 \mathrm{~h}$ of incubation, cell growth was determined in triplicate in three independent experiments. Bars, s.d. ${ }^{\star *}$ and *, differ from ACHN/P $(P<0.01$ and $P<0.05$, respectively). 
expression of phosphorylated S6 in ACHN/R cells could not be observed before and after temsirolimus treatment (Figure 4A).

As shown in Figure 4B, ACHN sublines exhibited varied patterns of activation status of Akt, p44/42 MAPK and STAT3 before and after temsirolimus treatment. Treatment with temsirolimus resulted in the marked downregulation of phosphorylated Akt in ACHN/P cells; however, the constitutive expression of phosphorylated Akt could be detected in ACHN/R cells following temsirolimus treatment. Despite the lack of a significant difference in phosphorylated p44/42 MAPK expression in ACHN/P cells before and after temsirolimus treatment, the expression level of phosphorylated p44/42 MAPK in ACHN/R cells, which was shown to be higher than that in ACHN/P cells, was further upregulated by treatment with temsirolimus. In both $\mathrm{ACHN}$ sublines, however, there were no significant differences in the expression levels of phosphorylated STAT3 before and after temsirolimus treatment.

Growth inhibitory effects of LY294002, U0126 and/or KU0063794 on ACHN sublines. The effects of additional treatment with sublethal doses of LY294002 and/or U0126, a selective inhibitor of phosphoinositol 3'-kinase (PI3K) and MAPK kinase, respectively, on the sensitivities of ACHN sublines to temsirolimus were evaluated. As shown in Figure 5A, the growth inhibitory effects of combined treatments with temsirolimus and

A

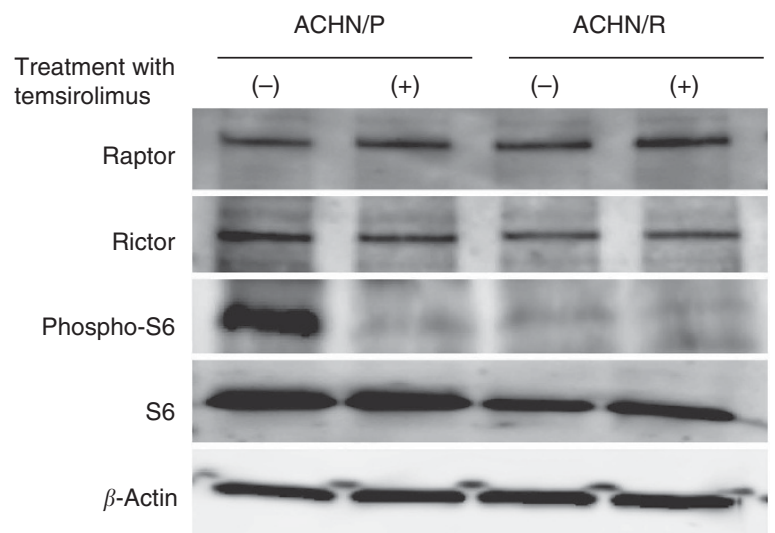

B

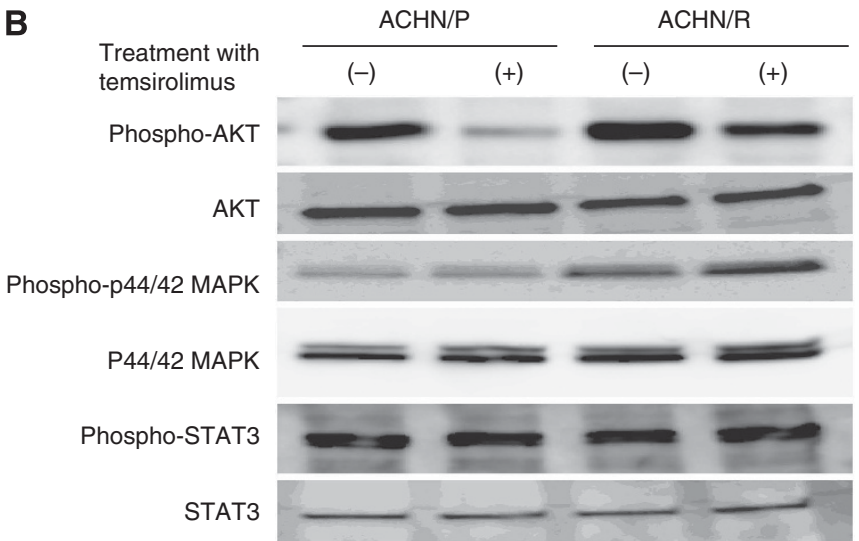

Figure 4. Changes in expression patterns of key molecules involved in signal transduction in $\mathrm{ACHN}$ sublines following treatment with temsirolimus. (A) Expression levels of raptor, rector, total and phosphorylated (phospho) S6 and $\beta$-actin in ACHN sublines before and after treatment with temsirolimus $(10 \mu \mathrm{M})$ were analysed using western blotting. (B) Expression levels of total and phosphorylated (phospho) Akt, p44/42 mitogen-activated protein kinase (MAPK) and signal transducers and activation of transcription 3 (STAT3) in ACHN sublines before and after treatment with temsirolimus $(10 \mu \mathrm{M})$ were analysed using western blotting. either LY294002 or U0126 on ACHN/R cells were weaker than those on ACHN/P cells, despite no additive impact of these combined therapies on the growth of ACHN/P cells; however, the combined treatment of $\mathrm{ACHN} / \mathrm{R}$ cells with temsirolimus, LY294002 and U0126 resulted in a degree of growth inhibition similar to that of ACHN/P.

We subsequently assessed the impact of inactivating mTORC1 and mTORC2 simultaneously using KU0063794, a dual inhibitor of mTORC1 and mTORC2, on the growth of ACHN sublines. As shown in Figure 5B, there was no significant difference in the sensitivity to KU0063794 between ACHN sublines.

Effects of temsirolimus treatment on the in vivo growth of ACHN sublines. To compare in vivo growth patterns of ACHN sublines with and without the administration of temsirolimus, $5 \times 10^{6}$ cells of each cell line were injected subcutaneously into 20 nude mice, and then they were randomly selected for treatment with either temsirolimus or the vehicle. As shown in Figure 6A, there was no significant difference between the volume changes of $\mathrm{ACHN} / \mathrm{P}$ and $\mathrm{ACHN} / \mathrm{R}$ tumours in mice receiving the vehicle. However, the administration of temsirolimus induced growth
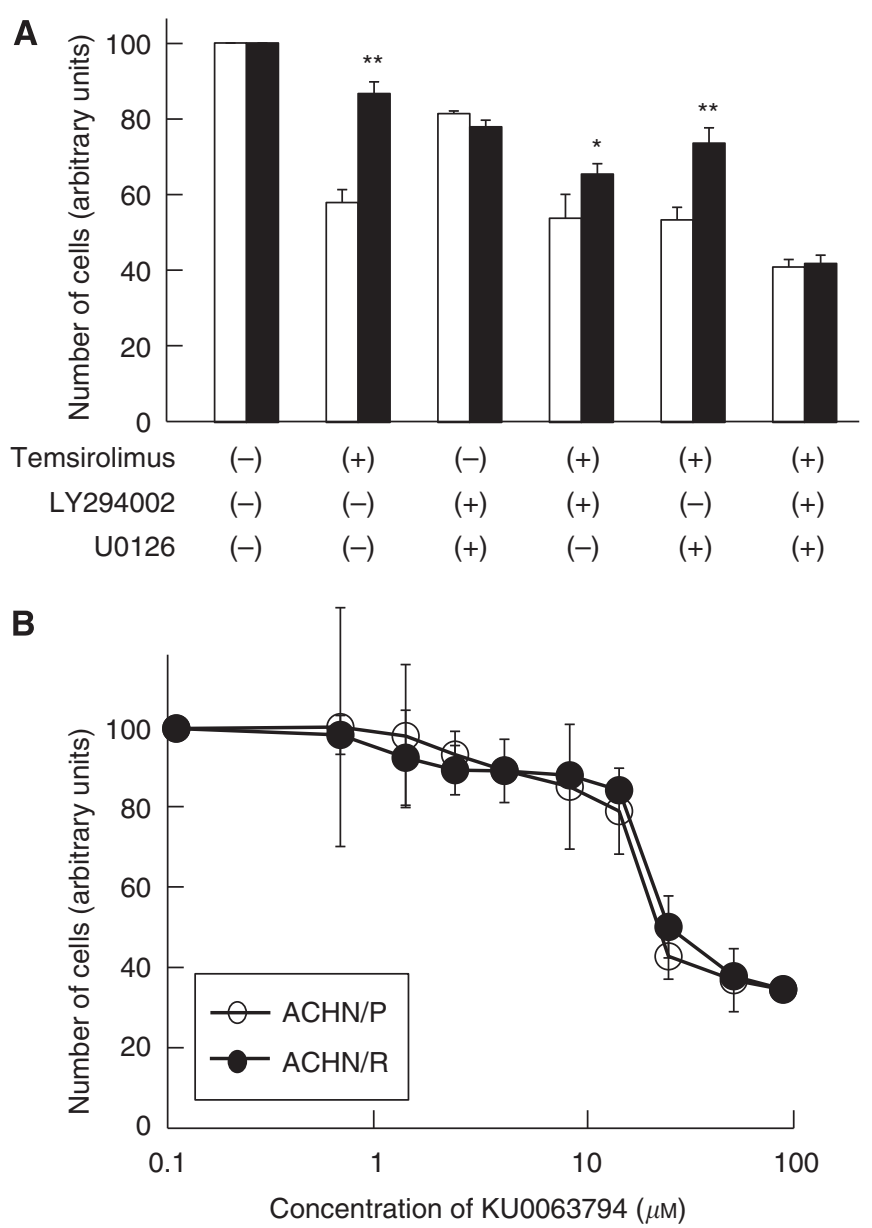

Figure 5. (A) Effects of combined treatment with temsirolimus, LY294002 and/or U0126 on the in vitro growth of ACHN sublines. Each cell line was treated with temsirolimus $(10 \mu \mathrm{M})$, LY294002 $(10 \mu \mathrm{M})$ and/or U0126 (10 $\mu \mathrm{m})$ and after incubation with these agents for $48 \mathrm{~h}$, cell numbers of each cell line were counted in triplicate. Bars, s.d. ** and *, differ from ACHN/P $(P<0.01$ and $P<0.05$, respectively). (B) Effect of treatment with KU0063794 on the in vitro cell growth of $\mathrm{ACHN}$ sublines. Each cell line was treated with the indicated doses of KU0063794. After $48 \mathrm{~h}$ of incubation, cell growth was determined in triplicate in three independent experiments. 

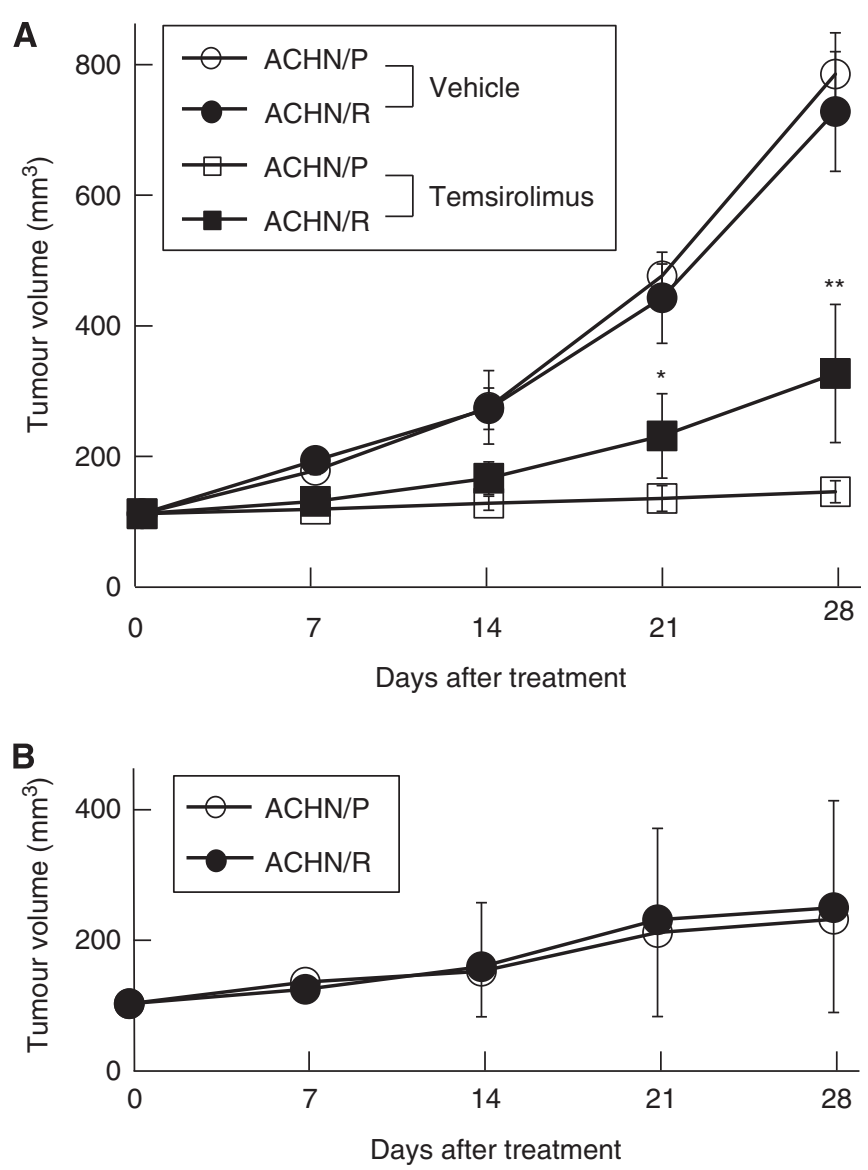

Figure 6. (A) Effect of treatment with temsirolimus on the in vivo growth of $\mathrm{ACHN}$ sublines. Twenty nude mice were subcutaneously given $5 \times 10^{6}$ cells of each $\mathrm{ACHN}$ subline, then randomly selected for treatment with either $10 \mathrm{mg} \mathrm{kg}^{-1}$ of temsirolimus or vehicle three times per week for 4 weeks, and the subcutaneous tumour volume was measured using calipers. Bars, s.d. ** and *, differ from ACHN/P $(P<0.01$ and $P<0.05$, respectively). (B) Effect of treatment with KU0063794 on the in vivo cell growth of $\mathrm{ACHN}$ sublines. Ten nude mice were subcutaneously given $5 \times 10^{6}$ cells of each ACHN subline, then treated with $8 \mathrm{mg} \mathrm{kg}^{-1}$ of KU0063794 twice per week for 4 weeks, and the subcutaneous tumour volume was measured using calipers. Bars, s.d.

inhibition of both $\mathrm{ACHN} / \mathrm{P}$ and $\mathrm{ACHN} / \mathrm{R}$ tumours, and the growth inhibitory effect of temsirolimus on the ACHN/P tumour was highly significant, compared with that on the ACHN/R tumour.

We then compared the in vivo effect of treatment with KU0063794 on ACHN sublines. KU0063794 markedly inhibited the growth of both ACHN/P and ACHN/R tumours, and there was no significant difference in the growth patterns between ACHN sublines.

\section{DISCUSSION}

The recent introduction of molecular targeted agents into clinical practice, which revolutionised the therapeutic strategy for patients with advanced RCC, has definitively improved the survival of these patients (Bellmunt et al, 2007; Lane et al, 2007); however, disease progression ultimately occurs in the majority of such patients during treatment with these agents (Rini and Atkins, 2009). To date, there have been few studies investigating the mechanisms mediating acquired resistance to molecular targeted drugs by RCC; accordingly, we developed a human RCC cell resistant to temsirolimus, a potent inhibitor of mTOR that is currently regarded as a first-line agent for patients with either poor-risk or non-clear cell advanced RCC (Voss et al, 2011), in order to characterise the changes in molecular profiles associated with the acquisition of a phenotype that is resistant to temsirolimus in this cell line.

The temsirolimus-resistant human RCC cell line, ACHN/R, generated in this study was shown to have an $\mathrm{IC}_{50}$ that was approximately sixfold higher than that of the parental cell line, $\mathrm{ACHN} / \mathrm{P}$. In most previous studies investigating the mechanism involved in drug resistance, a cell line showing extremely high resistance to the target drug compared with that employed in the present study was used (Guix et al, 2008; Muramaki et al, 2009). However, considering the concentration of each drug used during the development of such a highly resistant cell line, it might be more beneficial to select a cell line moderately resistant to the target drug, like ACHN/R cells, in order to achieve clinically relevant findings.

Then, we assessed whether ACRN/R cells have also acquired cross-resistance to molecular targeted agents other than temsirolimus. Although there were no significant differences in the sensitivities to TKIs, sorafenib and sunitinib between ACHN sublines, ACHN/R cells showed cross-resistance to another mTOR inhibitor, everolimus. Furthermore, we have recently developed an everolimus-resistant ACHN cell line, which also exhibited crossresistance to temsirolimus, but not to TKIs (data not shown), whereas we previously reported that a human RCC cell line resistant to sunitinib showed cross-resistance to sorafenib, but not to mTOR inhibitors (Sakai et al, 2013). These experimental findings indicate the usefulness of a sequential administration of mTOR inhibitor and TKI for advanced RCC; however, in a clinical setting, there have been several studies showing a favourable response to second-line TKI following the failure of initially administered TKI for patients with metastatic RCC (González Larriba et al, 2012). Therefore, it is necessary to further elucidate the mechanism underlying an acquired resistance to molecular targeted agents by employing both experimental and clinical approaches to generate conclusive findings concerning suitable sequential therapy for advanced RCC.

The involvement of apoptosis-related proteins in the acquisition of resistance to molecular targeted drugs has been suggested in various previous studies (Morgillo et al, 2007; Okamoto et al, 2012); hence, we also evaluated the changes in the expression of major apoptosis-related proteins in ACHN sublines with and without temsirolimus treatment. Despite the lack of differences in the expression of Bcl-xL and clusterin between ACHN sublines, temsirolimus treatment induced the upregulation of $\mathrm{Bcl}-2$ in $\mathrm{ACHN} / \mathrm{R}$ cells as well as that of Bax in ACHN/P cells. To date, several studies have presented findings that support the present outcomes (Majumder et al, 2004; Darwiche et al, 2011; Singh et al, 2012). For example, inactivation of the mTOR pathway by a protein kinase- $\delta$ inhibitor, rottlerin, caused apoptosis in human pancreatic cancer stem cells, accompanying the inhibition of Bcl-2 expression and induction of Bax (Singh et al, 2012), whereas enforced Bcl-2 overexpression in prostate epithelial cells inhibited apoptosis induced by an mTOR inhibitor, resulting in partial resistance to this agent (Majumder et al, 2004). Collectively, these findings suggest that the supression of anti-apoptotic proteins, such as Bcl-2, could be one of the promising approaches for overcoming resistance to mTOR inhibitors.

It is of interest to investigate differences in the statuses of signal transduction pathways between ACHN sublines with and without exposure to temsirolimus, as the persistent activation of major signal transduction pathways in the presence of targeted agents has been demonstrated to have crucial roles in the acquisition of a 
drug-resistant phenotype in several types of cancer cell (Okabe et al, 2009; Di Nicolantonio et al, 2010; Sakai et al, 2013). The expression levels of raptor and rictor were not changed following treatment with temsirolimus in ACHN sublines. Furthermore, treatment of ACHN/P cells with temsirolimus resulted in the complete inhibition of phosphorylated S6 expression; however, the S6 pathway in ACHN/R cells was completely inactivated before and after temsirolimus treatment. These findings strongly suggest the involvement of a signaling pathway different from that via mTORC1 in the survival of ACHN/R cells in the presence of temsirolimus.

As expected, according to the finding on the activated status of S6, Akt was constitutively activated in ACHN/R cells after treatment with temsirolimus, and the activation of p44/42 MAPK in ACHN/R cells, which was shown to be marked compared with that in ACHN/P cells, was further stimulated by treatment with temsirolimus. It has been well documented that despite the activation of the Akt signaling pathway by low concentrations of mTOR inhibitor through a negative feedback loop under the inhibition of mTORC1 signaling, higher doses of mTOR inhibitor inactivate Akt mainly via the mTORC2 signaling pathway (Chen et al, 2010), whereas in ACHN/R cells, higher dose of temsirolimus failed to inactivate Akt. As for changes in the activation status of p44/42 MAPK in ACHN sublines, it would be difficult to explain the overexpression and further temirolimusinduced upregulation of p44/42 MAPK in ACHN/R cells based on the established signaling cascade through mTOR. However, several recent studies have suggested an association between mTOR inhibition and activation of the MAPK signaling pathway (Carracedo et al, 2008; Chen et al, 2010). For example, treatment with an mTOR inhibitor was shown to activate p44/42 MAPK through a PI3K-dependent feedback loop in a prostate cancer model (Carracedo et al, 2008). Collectively, these findings suggest that continuous inhibition of mTORC1 during the development of ACHN/R markedly shifts the balance to increased mTORC2 activity for the survival of ACHN/R cells on exposure to temsirolimus.

The data on changes in the activation status of signal transduction pathways in ACHN sublines following treatment with temsirolimus provide a strong rationale for simultaneously targeting the activities of Akt and MAPK. In fact, additional treatment with either LY294002 or U0126 failed to restore the sensitivity of ACHN/R cells to temsirolimus to that of ACHN/P cells; however, the growth inhibitory effect of the combined treatment with temsirolimus, LY294002 and U0126 on ACHN/R cells appeared to be similar to that on ACHN/P cells. Furthermore, because of the complete inactivation of the signaling pathway via mTORC1 in ACHN/R cells, the continuous activation of Akt and MAPK in ACHN/R cells after treatment with temsirolimus could be expected to be exerted via the mTORC2 signaling pathway. Therefore, the cytotoxic effects of a dual inhibitor of mTORC1 and mTORC2, KU0063794, on ACHN sublines were compared, showing that there was no significant difference in sensitivities to KU0063794 between ACHN sublines both in vitro and in vivo. Considering these findings, maintaining activation of the signal transduction pathways, particularly the Akt and MAPK pathways, during temsirolimus treatment may be associated with the acquisition of a phenotype resistant to this agent in RCC cells, which may be overcome by agents capable of inactivating the signaling pathway via mTORC2. However, for elucidating the functional role of $\mathrm{mTORC} 2$ in the acquired resistance to temsirolimus, it would be eventually required to investigate whether the inactivation of mTORC2 alone re-sensitises ACHN/R cells to temsirolimus.

Acquired resistance to a targeted agent may develop accompanying modifications in various molecular events, including apoptosis, signal transduction and angiogenesis; therefore, the growth patterns of parental and resistant cells in vitro may not always reflect those in vivo. In our previous study, there was no significant difference in the in vivo growth inhibitory effects of sunitinib between parental and sunitinib-resistant RCC cell lines, despite the presence of extensive apoptosis in parental RCC tumours compared with that in sunitinib-resistant RCC tumours (Sakai et al, 2013). In this study, however, the therapeutic impact of treatment with temsirolimus on $\mathrm{ACHN} / \mathrm{P}$ tumours was significantly more favourable than that on ACHN/R tumours, like in the in vitro study. Therefore, the temsirolimus-resistant RCC model developed in this study could also be applied to investigate the mechanism mediating the acquisition of temsirolimus resistance in vivo.

In conclusion, we successfully developed $\mathrm{ACHN} / \mathrm{R}$, a human RCC cell line with a phenotype that is resistant to temsirolimus, which exhibited cross-resistance to everolimus, but not to sorafenib or sunitinib. We subsequently showed the upregulation of Bcl-2 in ACHN/R cells as well as that of Bax in ACHN/P cells following treatment with temsirolimus. Despite the complete inactivation of the mTORC1 signaling pathway in ACHN/R cells irrespective of temsirolimus treatment, the persistent phosphorylation of Akt and MAPK in the presence of temsirolimus was observed in ACHN/R cells, but not in ACHN/P cells. Furthermore, the resistance to temsirolimus in ACHN/R cells could be overcome by combined treatment with selective inhibitors of Akt and MAPK signaling pathways or a dual inhibitor of mTORC1 and mTORC2. Collectively, these findings suggest that maintaining activation of signal transduction via mTORC2, but not that via mTORC1, may be involved, at least in part, in the acquisition of a phenotype that is resistant to temsirolimus in RCC cells, and that it would be one of the promising approaches for overcoming temsirolimus resistance in patients with RCC to target the signaling pathways via mTORC2. However, it should be finally emphasised that this study was conducted using an RCC cell line, ACHN, alone; therefore, it would be necessary to investigate the mechanism of acquired resistance to temsirolimus in additional RCC cell lines to confirm the significance of currently achieved outcomes.

\section{REFERENCES}

Bellmunt J, Montagut C, Albiol S, Carles J, Maroto P, Orsola A (2007) Present strategies in the treatment of metastatic renal cell carcinoma: an update on molecular targeting agents. BJU Int 99: 274-280.

Carew JS, Kelly KR, Nawrocki ST (2011) Mechanisms of mTOR inhibitor resistance in cancer therapy. Target Oncol 6: 17-27.

Carracedo A, Ma L, Teruya-Feldstein J, Rojo F, Salmena L, Alimonti A, Egia A, Sasaki AT, Thomas G, Kozma SC, Papa A, Nardella C, Cantley LC, Baselga J, Pandolfi PP (2008) Inhibition of mTORC1 leads to MAPK pathway activation through a PI3K-dependent feedback loop in human cancer. J Clin Invest 118: 3065-3074.

Chen XG, Liu F, Song XF, Wang ZH, Dong ZQ, Hu ZQ, Lan RZ, Guan W, Zhou TG, Xu XM, Lei H, Ye ZQ, Peng EJ, Du LH, Zhuang QY (2010) Rapamycin regulates Akt and ERK phosphorylation through mTORC1 and mTORC2 signaling pathways. Mol Carcinog 49: 603-610.

Darwiche N, Sinjab A, Abou-Lteif G, Chedid MB, Hermine O, Dbaibo G, Bazarbachi A (2011) Inhibition of mammalian target of rapamycin signaling by everolimus induces senescence in adult T-cell leukemia/ lymphoma and apoptosis in peripheral T-cell lymphomas. Int J Cancer 129: 993-1004.

Di Nicolantonio F, Arena S, Tabernero J, Grosso S, Molinari F, Macarulla T, Russo M, Cancelliere C, Zecchin D, Mazzucchelli L, Sasazuki T, Shirasawa S, Geuna M, Frattini M, Baselga J, Gallicchio M, Biffo S, Bardelli A (2010) Deregulation of the PI3K and KRAS signaling pathways in human cancer cells determines their response to everolimus. J Clin Invest 120: 2858-2866.

Foster KG, Fingar DC (2010) Mammalian target of rapamycin (mTOR): conducting the cellular signaling symphony. J Biol Chem 285: 14071-14077. 
González Larriba JL, Espinosa E, García Carbonero I, García-Donas J, López M, Meana A, Puente J, Bellmunt J (2012) Sequential therapy in metastatic renal cell carcinoma: pre-clinical and clinical rationale for selecting a second- or subsequent-line therapy with a different mechanism of action. Cancer Metastasis Rev 31(Suppl 1): S11-S17.

Guix M, Faber AC, Wang SE, Olivares MG, Song Y, Qu S, Rinehart C, Seidel B, Yee D, Arteaga CL, Engelman JA (2008) Acquired resistance to EGFR tyrosine kinase inhibitors in cancer cells is mediated by loss of IGF-binding proteins. J Clin Invest 118: 2609-2619.

Hudes G, Carducci M, Tomczak P, Dutcher J, Figlin R, Kapoor A, Staroslawska E, Sosman J, McDermott D, Bodrogi I, Kovacevic Z, Lesovoy V, Schmidt-Wolf IG, Barbarash O, Gokmen E, O’Toole T, Lustgarten S, Moore L, Motzer RJ (2007) Temsirolimus, interferon alfa, or both for advanced renal-cell carcinoma. $N$ Engl J Med 356: 2271-2281.

Kumano M, Miyake H, Kurahashi T, Yamanaka K, Fujisawa M (2008) Enhanced progression of human prostate cancer PC 3 cells induced by the microenvironment of the seminal vesicle. Br J Cancer 98: 356-362.

Lane BR, Rini BI, Novick AC, Campbell SC (2007) Targeted molecular therapy for renal cell carcinoma. Urology 69: 3-10.

Le Tourneau C, Faivre S, Serova M, Raymond E (2008) mTORC1 inhibitors: is temsirolimus in renal cancer telling us how they really work? $\mathrm{Br} J$ Cancer 99: 1197-1203.

Majumder PK, Febbo PG, Bikoff R, Berger R, Xue Q, McMahon LM, Manola J, Brugarolas J, McDonnell TJ, Golub TR, Loda M, Lane HA, Sellers WR (2004) mTOR inhibition reverses Akt-dependent prostate intraepithelial neoplasia through regulation of apoptotic and HIF-1-dependent pathways. Nat Med 10: 594-601.

Menon S, Manning BD (2008) Common corruption of the mTOR signaling network in human tumors. Oncogene 27(Suppl 2): S43-S51.

Miyake H, Kurahashi T, Takenaka A, Inoue TA, Fujisawa M (2009) Clinical outcome of combined immunotherapy with interferon-alpha and low-dose interleukine-2 for Japanese patients with metastatic renal cell carcinoma. Urol Oncol 27: 598-603.

Morgillo F, Bareschino MA, Bianco R, Tortora G, Ciardiello F (2007) Primary and acquired resistance to anti-EGFR targeted drugs in cancer therapy. Differentiation 75: 788-799.

Muramaki M, So A, Hayashi N, Sowery R, Miyake H, Fujisawa M, Gleave ME (2009) Chemosensitization of gemcitabine-resistant human bladder cancer cell line both in vitro and in vivo using antisense oligonucleotide targeting the anti-apoptotic gene, clusterin. BJU Int 103: 384-390.
Okabe T, Okamoto I, Tsukioka S, Uchida J, Hatashita E, Yamada Y, Yoshida T, Nishio K, Fukuoka M, Jänne PA, Nakagawa K (2009) Addition of S-1 to the epidermal growth factor receptor inhibitor gefitinib overcomes gefitinib resistance in non-small cell lung cancer cell lines with MET amplification. Clin Cancer Res 15: 907-913.

Okamoto K, Okamoto I, Hatashita E, Kuwata K, Yamaguchi H, Kita A, Yamanaka K, Ono M, Nakagawa K (2012) Overcoming erlotinib resistance in EGFR mutation-positive non-small cell lung cancer cells by targeting survivin. Mol Cancer Ther 11: 204-213.

Parton M, Gore M, Eisen T (2006) Role of cytokine therapy in 2006 and beyond for metastatic renal cell cancer. J Clin Oncol 24: 5584-5592.

Rini BI, Atkins MB (2009) Resistance to targeted therapy in renal-cell carcinoma. Lancet Oncol 10: 992-1000.

Rini BI, Rathmell WK, Godley P (2008) Renal cell carcinoma. Curr Opin Oncol 20: 300-306.

Sakai I, Miyake H, Fujisawa M (2013) Acquired resistance to sunitinib in human renal cell carcinoma cells is mediated by constitutive activation of signal transduction pathways associated with tumour cell proliferation. BJU Int 112(2): E211-E220.

Singh BN, Kumar D, Shankar S, Srivastava RK (2012) Rottlerin induces autophagy which leads to apoptotic cell death through inhibition of PI3K/ Akt/mTOR pathway in human pancreatic cancer stem cells. Biochem Pharmacol 84: 1154-1163.

Sparks CA, Guertin DA (2010) Targeting mTOR: prospects for mTOR complex 2 inhibitors in cancer therapy. Oncogene 29: 3733-3744.

Terakawa T, Miyake H, Furukawa J, Ettinger SL, Gleave ME, Fujisawa M (2009) Enhanced sensitivity to androgen withdrawal due to overexpression of interleukin-6 in androgen-dependent human prostate cancer LNCaP cells. Br J Cancer 101: 1731-1739.

Voss MH, Molina AM, Motzer RJ (2011) mTOR inhibitors in advanced renal cell carcinoma. Hematol Oncol Clin North Am 25: 835-852.

This work is published under the standard license to publish agreement. After 12 months the work will become freely available and the license terms will switch to a Creative Commons AttributionNonCommercial-Share Alike 3.0 Unported License. 